\title{
Matter Wave Interferometry of a Levitated Thermal Nano-Oscillator Induced and Probed by a Spin
}

\author{
M. Scala, ${ }^{1}$ M. S. Kim, ${ }^{2}$ G. W. Morley, ${ }^{3}$ P. F. Barker,${ }^{1}$ and S. Bose $^{1}$ \\ ${ }^{1}$ Department of Physics and Astronomy, University College London, \\ Gower St., London WC1E 6BT, United Kingdom \\ ${ }^{2} Q O L S$, Blackett Laboratory, Imperial College London, London SW7 2BW, United Kingdom \\ ${ }^{3}$ Department of Physics, University of Warwick, \\ Gibbet Hill Road, Coventry CV4 7AL, United Kingdom
}

(Dated: June 4, 2018)

\begin{abstract}
We show how the interference between spatially separated states of the center of mass (COM) of a mesoscopic harmonic oscillator can be evidenced by coupling it to a spin and performing solely spin manipulations and measurements (Ramsey Interferometry). We propose to use an optically levitated diamond bead containing an NV center spin. The nano-scale size of the bead makes the motional decoherence due to levitation negligible. The form of the spin-motion coupling ensures that the scheme works for thermal states so that moderate feedback cooling suffices. No separate control or observation of the COM state is required and thereby one dispenses with cavities, spatially resolved detection and low mass-dispersion ensembles. The controllable relative phase in the Ramsey interferometry stems from a gravitational potential difference so that it uniquely evidences coherence between states which involve the whole nano-crystal being in spatially distinct locations.
\end{abstract}

Quantum mechanics does not itself provide any limits to its applicability. Its formalism is independent of mass - an isolated object, however massive, should exhibit superpositions. While it is important to test whether this is true for a mesoscopic object, such tests face the obstacle of decoherence. Decoherence, caused by the coupling of a system to its environment, causes a reduction in the visibility of interference patterns that evidence superpositions [1, 2]. Fundamental modifications of quantum behavior may add to this $[3-5]$. The pragmatic approach would be to better isolate mesoscopic objects and engineer quantum behavior. This has been happening successfully - a superconducting system originally invoked to probe the limitations of quantum behavior [6], is now used as a qubit [7]. Matter wave interferometry with macromolecules has extended the quantum realm [8]. Further extension may be possible by coupling nanocantilevers to photons [9, 10], or qubits 11, 12]. Optical counterparts have also been explored [13]. A nano-scale object levitated by a (classical) light field can be fruitful for demonstrations of quantum behavior 14 16].

In this letter we first enlist five desiderata which would simplify the path to extend quantum mechanics to larger objects and then propose a Ramsey interferometry experiment on a spin-mechanical oscillator hybrid system that meets these desiderata. Firstly, it is challenging to create nearly "pure" non-classical states of the center of mass (COM) of mesoscopic objects, requiring cooling of the object to its ground state, though there is much work in this direction [15-20]. It would thus be very useful to be able to test the validity of the superposition principle with the mechanical oscillator initially in thermal equilibrium with its environment (this is our desideratum 1). Secondly, in many proposals, a high finesse cavity facilitates the preparation and probing of nonclassical states

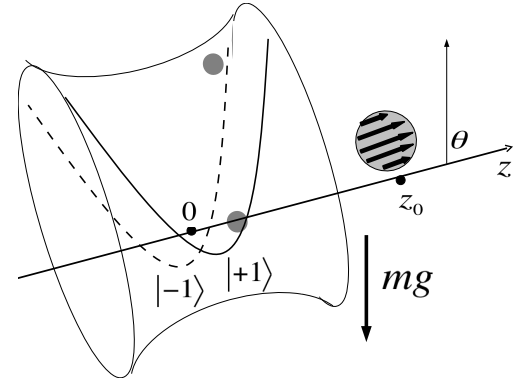

FIG. 1: Setup: An optical trap holds a diamond bead with an NV center with both weakest confinement and spin quantization along the $\mathrm{z}$ axis. A magnetized sphere at $z_{0}$ produces spin-dependent shifts to the center of the harmonic well. An angle $\theta$ between the vertical and the $z$ axis places the centers of the wells corresponding to the $|+1\rangle$ and $|-1\rangle$ states in different gravitational potentials. A random coherent state of the center of mass of the bead oscillates as different coherent states in the two wells (grey filled circles), accumulating a relative gravitational phase difference due to superpositions. At $t_{0}=2 \pi / \omega_{z}$ this phase can be read from spin measurements.

of a mechanical object [9, 10, 19, 20] (note, however, an exception [21]). However, it is demanding for a cavity coupled to a mechanical resonator to have a high finesse 22]. Time resolutions in measurements of cavity fields is also limited (although, see [18]). Thus avoiding a cavity will form our desideratum 2. Matter-wave experiments satisfy desiderata 1 and 2 , but require the detection of a spatial interference pattern generated by an ensemble of particles [8]. Keeping this pattern spatially resolvable and robust to mass and velocity spreads of the ensemble is challenging - a challenge that would probably partially carry over to some optomechanics [19, 20] and spin-optomechanics 23] schemes. Avoiding ensem- 
bles and spatially resolved measurements will thus form our desiderata 3 and 4. Lastly, controlling an interference pattern by imparting a tunable relative phase between superposed paths is the "litmus test" of any interference - yet this has been largely overlooked in the context of mesoscopic mechanical oscillators. The controllable relative phase should stem from a field such as gravity that only interacts with the mass and has no chance to directly interact with auxillary probes such as the spin. In this way, by detecting the influence of gravity on the interference of spin, one can be sure that the mechanical object had been part of a quantum superposition during an experiment. Proposals so far have resorted to subtle methodology such as detecting a mass dependent partial decoherence of a probe 9, 24] or a periodic decoherencerecoherence dynamics of a probe 9 -11, 24, 25] (this is susceptible to temperature [26]). The requirement of a more transparent interference experiment using a "controllable relative phase" between different COM states of a mechanical oscillator, one which can be solely imparted to the oscillator, yet can be read from a probe, will thus form our desideratum 5 . We now present a scheme that satisfy all the 5 desiderata.

Our setup is shown in Fig, 1 and consists of a nanoscale diamond bead containing a single spin- $1 \mathrm{NV}$ center levitated by an optical tweezer in ultra-high vacuum. We need a static magnetic field gradient to couple the motion of the bead in the harmonic potential of the tweezer to the $S=1$ spin of the NV center. This can be generated by a magnetized sphere with a permanent dipole moment $\mathbf{m}=\left(0,0, m_{z}\right)$ oriented along the $z$ direction. Let the centers of the harmonic potential and the magnetized sphere be at $(0,0,0)$ and $\left(0,0, z_{0}\right)$ respectively. Expanding the magnetic field of the sphere around $(0,0,0)$, we get

$$
B_{x}=-B_{0} x, \quad B_{y}=-B_{0} y, \quad B_{z}=\frac{\mu_{0} m_{z}}{2 \pi\left|z_{0}\right|^{3}}+2 B_{0} z,
$$

where $B_{0}=3 \mu_{0} m_{z} /\left(4 \pi z_{0}^{5}\right)$. Therefore the Hamiltonian describing the interaction between the spin of the NV center and the vibrational motion can be written as:

$H_{\mathrm{int}}=-\lambda\left[2 S_{z}\left(c+c^{\dagger}\right)-\sqrt{\frac{\omega_{z}}{\omega_{x}}} S_{x}\left(a+a^{\dagger}\right)-\sqrt{\frac{\omega_{z}}{\omega_{y}}} S_{y}\left(b+b^{\dagger}\right)\right]$,

where

$$
\lambda=\frac{3 \mu_{0} m_{z} z_{0}}{4 \pi\left|z_{0}\right|^{5}} g_{N V} \mu_{B} \sqrt{\frac{\hbar}{2 m \omega_{z}}},
$$

$m$ being the mass of the bead, $g_{N V}$ the Lande factor of the NV center and $\mu_{B}$ the Bohr magneton. To this we must add the free Hamiltonian of the NV center and of the bead motion, i.e. $H_{\text {free }}=D S_{z}^{2}+\hbar\left(\omega_{x} a^{\dagger} a+\omega_{y} b^{\dagger} b+\right.$ $\left.\omega_{z} c^{\dagger} c\right)$. The anisotropy $D S_{z}^{2}$ of the NV center is not a problem as the possibility to align the $z$ axis of the defect in the nano-diamond to any desired direction has been recently demonstrated [27, 28]. We consider the Zeeman splitting of $|+1\rangle$ and $|-1\rangle$ due to the zeroth order expansion of $B_{z}$ to be cancelled by addition of a uniform magnetic field along $z$.

Finally, we assume $\omega_{x}, \omega_{y} \gg \omega_{z}$ and add the interaction with the Earth's gravitational field $m g z \cos \theta$, where $\theta$ is the angle between the $z$ direction and the free fall acceleration (see Fig. 1). Therefore we obtain the Hamiltonian:

$$
H=D S_{z}^{2}+\hbar \omega_{z} c^{\dagger} c-2\left(\lambda S_{z}+\Delta \lambda\right)\left(c+c^{\dagger}\right),
$$

with

$$
\Delta \lambda=\frac{1}{2} m g \cos \theta \sqrt{\frac{\hbar}{2 m \omega_{z}}} .
$$

The Hamiltonian above represents a harmonic oscillator whose center depends on the eigenvalue of $S_{z}$. For each of $S_{z}=-1,0$, and +1 , we can calculate the evolution of the oscillator, when its initial state is a coherent state $|\beta\rangle$ of the harmonic well centered at $z=0$. Transforming by means of an appropriate displacement operator, we can calculate the evolution governed by a new Hamiltonian corresponding to a free harmonic oscillator with no displacement. Transforming back to the original representation, we get the three spin-dependent evolutions

$$
|\beta\rangle\left|s_{z}\right\rangle \rightarrow\left|\beta\left(t, s_{z}\right)\right\rangle\left|s_{z}\right\rangle
$$

where, denoting $u=2\left(s_{z} \lambda-\Delta \lambda\right) / \hbar \omega_{z}$,

$$
\begin{aligned}
\left|\beta\left(t, s_{z}\right)\right\rangle= & \mathrm{e}^{-\frac{i}{\hbar}\left(D-\hbar \omega_{z} u^{2}\right) t} \mathrm{e}^{i u^{2} \sin \left(\omega_{z} t\right)} \\
& \times\left|(\beta-u) \mathrm{e}^{-i \omega_{z} t}+u\right\rangle,
\end{aligned}
$$

with the ket on the right hand side being a coherent state and $s_{z}$ being an eigenvalue of $S_{z}$. The above time evolution is illustrated in phase space for two distinct values of $\beta$ in Fig 2, A striking feature of this evolution is that at time $t_{0}=2 \pi / \omega_{z}$ the oscillator state returns to its original coherent state $\beta$, for any $\beta$ and $s_{z}$. This feature implies that spin measurements at $t_{0}$ will be unaffected by any randomness in the initial motional state of the oscillator. We now exploit this key feature to propose a Ramsey interferometric scheme that does not require any preparation of the initial COM state, yet detects a phase difference stemming from superpositions involving distinct COM positions.

Ramsey interferometry - Consider that the system starts from a separable state $|\beta\rangle\left|s_{z}=0\right\rangle$. The first step applies a microwave pulse corresponding to the Hamiltonian $H_{m w}=\hbar \Omega(|+1\rangle\langle 0|+|-1\rangle\langle 0|+$ h.c. $)$, with $\Omega$ much larger than any other coupling constant, so that we can neglect any other interaction when applying the pulse. With the pulse duration $t_{p}=\pi /(2 \sqrt{2} \Omega)$, the spin state becomes a superposition with equal amplitudes of $|+1\rangle$ and $|-1\rangle:|\Psi(0)\rangle=|\beta\rangle\left(\frac{|+1\rangle+|-1\rangle}{\sqrt{2}}\right)$, which we take as 


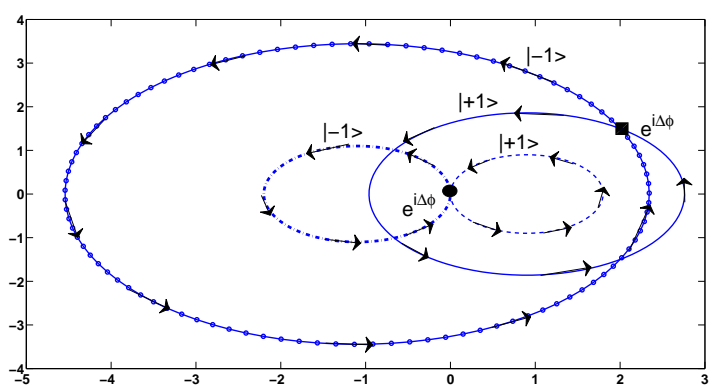

FIG. 2: The phase space evolution of two arbitrary initial coherent states $\beta=0$ (filled circle) and $\beta=2+i 1.5$ (filled square) with $\lambda=0.5 \hbar \omega_{z}$ and $\Delta \lambda=0.05 \hbar \omega_{z}$. The arrows show the direction of evolution of the coherent states with increasing time for a whole time period $t_{0}=2 \pi / \omega_{z}$. The return of each component to the initial coherent state at $t_{0}$ is evident. A relative phase $e^{i \Delta \phi_{\text {Grav }}}$ appears between the $|+1\rangle$ and $|-1\rangle$ component due to the gravitational potential difference between the centers of the phase space trajectories for $|-1\rangle$ (more towards the left of the figure) and $|+1\rangle$ (more towards the right of the figure).

the initial state for the interaction under the Hamiltonian (4). At the interaction time $t$, the state is then

$$
|\Psi(t)\rangle=\left(\frac{|\beta(t,+1)\rangle|+1\rangle+|\beta(t,-1)\rangle|-1\rangle}{\sqrt{2}}\right),
$$

which is the superposition we intend to evidence. Note from the expressions of $|\beta(t, \pm 1)\rangle$ in Eq.(7) that separated coherent states are involved in the above superposition along with phases due to gravitational potential, which are finally going to evidence the above superposition. The state after an oscillation period $t_{0}=2 \pi / \omega_{z}$ is

$$
\left|\Psi\left(t_{0}\right)\right\rangle=|\beta\rangle\left(\frac{|+1\rangle+\mathrm{e}^{i \Delta \phi_{\mathrm{Grav}}}|-1\rangle}{\sqrt{2}}\right),
$$

where, after dropping a global phase factor, we have

$$
\Delta \phi_{\mathrm{Grav}}=\frac{16 \lambda \Delta \lambda}{\hbar^{2} \omega_{z}} t_{0}
$$

To reveal $\Delta \phi_{\text {Grav }}$ we apply $H_{m w}$ again. After a time $t_{p}$, the population of the spin state with $S_{z}=0$ is:

$$
P_{0}\left(t=t_{0}+t_{p}\right)=\cos ^{2}\left(\frac{\Delta \phi_{\text {Grav }}}{2}\right),
$$

which gives a direct connection between the value of the phase shift and spin population. As $\Delta \phi_{\text {Grav }} \propto g$ (gravitational acceleration) can never appear as a relative phase between spin states unless spatially separated states of the COM were involved in the superposition $(|\beta(t,+1)\rangle|+1\rangle+|\beta(t,-1)\rangle|-1\rangle) / \sqrt{2}$ for $0<t<t_{0}$, the detection of $\Delta \phi_{\text {Grav }}$ evidences such a superposition.
Thermal effects - We can exploit the facts that the results given above are independent of the amplitude $\beta$ (see Fig 2] for an illustration) and that any thermal state $\rho_{t h}$ of the motion can be written as $\rho_{t h}=\int \mathrm{d}^{2} \beta P_{t h}(\beta)|\beta\rangle\langle\beta|$, where $P_{t h}$ is the Glauber $P$ representation for the thermal state. Considering the following initial state for the composite system:

$$
\rho(t=0)=\rho_{t h} \otimes \frac{1}{2}(|+1\rangle+|-1\rangle)(\langle+1|+\langle-1|),
$$

at time $t_{0}$ we have

$$
\begin{aligned}
\rho\left(t_{0}\right) & =\int \mathrm{d}^{2} \beta P_{t h}(\beta)|\beta\rangle\langle\beta| \\
& \otimes \frac{1}{2}\left(|+1\rangle+\mathrm{e}^{i \Delta \phi_{\text {Grav }}}|-1\rangle\right)\left(\langle+1|+\mathrm{e}^{-i \Delta \phi_{\text {Grav }}}\langle-1|\right),
\end{aligned}
$$

which shows that, after one period, the state of the system is again factorizable and that the phase difference accumulated by the spin states is not affected by the thermal motion. Basically, though a mixture of several Schrödinger cats $|\beta(t,+1)\rangle|+1\rangle+|\beta(t,-1)\rangle|-1\rangle$ is generated for $0<t<t_{0}$, the interference between the components $|\beta(t,+1)\rangle|+1\rangle$ and $|\beta(t,-1)\rangle|-1\rangle$ of the cat is independent of $\beta$. This immunity of the interference to thermal states hinges on the mass being trapped in a harmonic potential. We assume that anharmonic effects of the trapping potential will be avoided by feedback cooling of our oscillator to $\mathrm{mK}$ temperatures [30,31]. We will also justify that the heating during evolution is negligible for $0 \leq t \leq t_{0}$.

Experimental parameters - We now give the parameters necessary to obtain a good visibility of the interferometric fringes in a setup in which we are allowed to vary the angle $\theta$. As realistic values, we consider $\omega_{z} \sim 100 \mathrm{kHz}$ and diamond spheres whose radius $R \sim 100 \mathrm{~nm}$, so that, considering the density $3000 \mathrm{~kg} / \mathrm{m}^{3}$ for diamond, the corresponding mass is $\sim 1.25 \times 10^{-17} \mathrm{~kg}$. A good visibility of interferometry fringes in the population in Eq. (11) is given for $K=8 \lambda \Delta \lambda t_{0} /\left(\hbar^{2} \omega_{z} \cos \theta\right) \sim 10$, which makes the value of the population change completely from 0 to 1 when $\theta$ varies between $\pi / 2-\pi / 20$ and $\pi / 2$ (the $z$ axis is horizontal for $\theta=\pi / 2$ ). Assuming that the magnetic field in Eq. (1) is generated by a magnetized sphere with radius $r_{0}=40 \mu \mathrm{m}$ and magnetization $M=1.5 \times 10^{6} \mathrm{~A} / \mathrm{m}$ (typical for commercial magnets), and $z_{0}=120 \mu \mathrm{m}$, we get, according to $m_{z}=M \cdot(4 \pi / 3) r_{0}^{3}$ and Eqs. (3) and (5), the desired value of $K$.

Corresponding to such values, it is worth noting that the maximum separation between the coherent states involved in the interferometric scheme is, according to Eq. (6), $4 \lambda / \hbar \omega_{z} \simeq 0.03$, which shows that, during the scheme proposed, the superpositions involved are not macroscopic. This can be advantageous since it allows to have very small values for the motional decoherence rates, whose maximum value will be $\gamma_{s c} \cdot\left|2 \lambda / \hbar \omega_{z}\right|^{2}$, where $\gamma_{s c}$ is the decay rate associated with photon scattering from 
the trapping laser [16]. For the diamonds used in the laboratory, with a dielectric permittivity $\epsilon=1.5$, we have $\gamma_{s c} / \omega_{z}=\left(16 \pi^{3} / 15\right)((\epsilon-1) /(\epsilon+2))\left(R^{3} / \lambda_{0}^{3}\right) \simeq 5 \times 10^{-3}$, for spheres of radius $R=100 \mathrm{~nm}$ and trapping wavelength $\lambda_{0} \sim 1 \mu \mathrm{m}$. Thus the values we found allow us to neglect the decoherence due to light scattering for the duration $t_{0} \sim 10 \mu \mathrm{s}$ of our scheme. For the same reason heating, in the form of random momentum kicks during the $t_{0} \sim 10 \mu$ s duration, can be neglected. Moreover, feedback cooling to mK temperatures, which has already been demonstrated [30, 31], makes the thermal state phonon number of the levitated diamond to be $\sim 1000$, which is well below the the energy before which the harmonic approximation starts to break down. Thus our protocol should work even under a thermal environment.

The detrimental effects on the scheme are therefore only due to the dephasing of the NV center due to other spins in the diamond lattice. The value for this time $\left(T_{2}\right)$ in bulk diamond is exceptional ( $\sim$ ms [32]). While such a value would pose no dephasing in the timescale of our scheme, for $R \sim 20 \mathrm{~nm}$ nano-diamonds $T_{2} \sim 10 \mu \mathrm{s}$ is significantly lower due to interactions between the spin and defects on the surface 29]. Our larger (100 nm) beads would remove the surface further from the NV center and improve the coherence. Additionally, we propose to use to spin echo techniques along with a sudden change of the orientation (angle $\theta$ ) of the experimental setup, which is now experimentally feasible 33. If after one period $t_{0}$ we only apply a mw pulse so that the spin state $|+1\rangle$ goes to the state $|-1\rangle$ and vice versa, at time $t=2 t_{0}$ the random phases (at the origin of dephasing) acquired by the spin states during the second oscillation will cancel the ones acquired during the first oscillation, minimizing the dephasing effects. However, the spin echo pulse would also cause the spin states to acquire opposite values for the gravitational phases during the second period, cancelling out the phases accumulated after the first oscillation. This problem persists even if the refocusing pulse is orthogonal to the preparation pulse as in the CPMG experiment, where the sequence of pulses $(\pi / 2)_{x}-\left[\pi_{y}-\text { echo }\right]_{\text {repeat }}$ is used [29]. To avoid this, it is enough to reverse the direction of the $z$ axis with respect to the horizontal plane, so that $\theta \rightarrow \pi-\theta$, which corresponds to having the same evolutions as in Eq. (6) with the substitution $\Delta \lambda \rightarrow-\Delta \lambda$ : consequently, at time $t=2 t_{0}$, the phase difference acquired between $|-1\rangle$ and $|+1\rangle$ will be twice the one given in Eq. (10). The detection scheme can then proceed as described before, with an improved visibility. For our parameters [29], $t_{0} / T_{2} \sim 1$, we still expect a visible fringe, though its contrast is reduced to $1 / e \sim 0.36$.

Our proposal combines desirable aspects in a feasible experiment while some of individual elements have been studied before. For example, the ability to map the decoherence of a mesoscopic oscillator to a probe field [9] or qubit [24] has been appreciated before, as well as its possibility for a thermal state. In Ref. [34], the mapping of a geometric phase to a probe qubit has been studied - here we are exploiting a similar idea, but, instead, mapping a dynamical phase from a gravitational potential term in the Hamiltonian. The dynamics entailed by Eqs. (7) has been exploited in several papers [9 11, 23 25], though not for Ramsey interferometry. Similarly, levitated diamonds containing an NV center have very recently been considered for generating pure Schrödinger cats [23] (in fact, while the work of this paper was in progress). We differ from this scheme in terms of the absence of a cavity and the gravitationally induced Ramsey interferometry. Thus our scheme is easy to probe without having to read out the motional state. Indeed this latter aspect is the central advance of our scheme over previous works as it allows a spin probe (which can be measured fast) to accumulate a gravitational phase shift. Perhaps other applications of the qubit-oscillator coupling $S_{z}\left(c+c^{\dagger}\right)$ 34, 35] can also be studied with our setup.

Macroscopic limits of quantum superpositions can be reached in two ways - larger masses and larger spatial separations between the superposed components. Here we will be able to test quantum superpositions for a large mass $\left(\sim 10^{-17} \mathrm{~kg}\right)$, but the physical separation between the COM parts of the superposed states $|\beta(t,+1)\rangle|+1\rangle$ and $|\beta(t,-1)\rangle|-1\rangle$ is quite small (distance $\sim 0.03$ in phase space which is about 0.15 picometers; it can be increased possibly to about $\sim 3$ in phase space by decreasing $z_{0}$ to $1 \mu \mathrm{m}$ and $r_{0} \sim 1 / 3 \mu \mathrm{m}$ by using nano-magnets). However, that $\Delta \phi_{\text {Grav }}$ is still accumulated and significant to give a full fringe $\left(P_{0}\left(t=t_{0}+t_{p}\right)\right.$ ranging from 0 to 1 as $\theta$ is varied) is interesting. Though in the end we only measure the spin, it still evidences the involvement of the COM in superpositions of the hybrid system as it is impossible for a spin to obtain a gravitational phase shift on its own. Our proposal satisfies a few desiderata (modest cooling, no cavities, ensembles or spatially resolved measurements, Ramsey interferometry with controllable phase) which potentially makes the path to extend quantum mechanics to more macroscopic objects simpler.

We acknowledge the EPSRC grant EP/J014664/1 with which MS is funded. GWM is supported by the Royal Society. MSK acknowledges support of the Qatar National Research Fund (Grant NPRP 4-554-D84). We are very thankful to Hendrik Ulbricht and Andreas Schell for comments which have improved the manuscript.

[1] A. O. Caldeira and A. J. Leggett, Annals of Physics 149, 374 (1983).

[2] W. H. Zurek, Phys. Today 44 (10): 36, (1991).

[3] G. C. Ghirardi, A. Rimini, and T. Weber, Phys. Rev. D 34, 470 (1986); P. Pearle, Phys. Rev. A39, 2277 (1989).

[4] R. Penrose, General Relativity and Gravitation, 28, 581 
(1996).

[5] A. Bassi, K. Lochan, S. Satin, T. P. Singh, and H. Ulbricht, Rev. Mod. Phys. 85, 471 (2013).

[6] A. J. Leggett, A. Garg, Phys. Rev. Lett. 54, 857 (1985).

[7] J. R. Friedman, V. Patel, W. Chen, S. K. Tolpygo \& J. E. Lukens, Nature 406, 43 (2000).

[8] S. Gerlich, S. Eibenberger, M. Tomandl, S. Nimmrichter, K. Hornberger, P. J. Fagan, J. Tüxen, M. Mayor, and M. Arndt, Nature Comm. 2, 263 (2011).

[9] S. Bose, K. Jacobs, and P. L. Knight, Phys. Rev. A 59, 3204 (1999).

[10] W. Marshall, C. Simon, R. Penrose, and D. Bouwmeester, Phys. Rev. Lett., 91130401 (2003).

[11] A.D. Armour, M.P. Blencowe, and K. Schwab, Phys. Rev. Lett. 88, 148301 (2002).

[12] P. Rabl, P. Cappellaro, G. Dutt, L. Jiang, J. R. Maze, and M. D. Lukin, Phys. Rev. B 79, 041302 (2009).

[13] N. Bruno, A. Martin, P. Sekatski, N. Sangouard, R. Thew, and N. Gisin, arXiv:1212.3710 [quant-ph].

[14] P. F. Barker and M. N. Schneider, Phys. Rev. A 81, 023826 (2010).

[15] O. Romero-Isart, M. L. Juan, R. Quidant, and J. I. Cirac, New J. Phys. 12, 033015 (2010).

[16] D. E. Chang, C. A. Regal, S. B. Papp, D. J. Wilson, J. Ye, O. Painter, H. J. Kimble and P. Zoller, Proc. Natl. Acad. Sci. USA 107, 1005 (2010).

[17] M. Paternostro, D. Vitali, S. Gigan, M. S. Kim, C. Brukner, J. Eisert, M. Aspelmeyer, Phys. Rev. Lett. 99, 250401 (2007).

[18] M. R. Vanner, I. Pikovski, G. D. Cole, M. S. Kim, C. Brukner, K. Hammerer, G. J. Milburn, M. Aspelmeyer, Proc Natl Acad Sci USA 108, 16182 (2011).

[19] O. Romero-Isart, A. C. Panzer, F. Blaser, R. Kaltenbaek, N. Kiesel, M. Aspelmeyer, and J. I. Cirac, Phys. Rev. Lett. 107, 020405 (2011).

[20] O. Romero-Isart, Phys. Rev. A 84, 052121 (2011).

[21] B. Pepper, R. Ghobadi, E. Jeffrey, C. Simon, D. Bouwmeester, Phys. Rev. Lett. 109, 023601 (2012).

[22] D. Kleckner, W. T. M. Irvine, S. S. R. Oemrawsingh and D. Bouwmeester, Physical Review A 81, 043814 (2010).

[23] Zhang-qi Yin, Tongcang Li, Xiang Zhang, L. M. Duan, arXiv:1305.1701.

[24] S. Bose, Phys. Rev. Lett. 96, 060402 (2006).

[25] O. Romero-Isart, L. Clemente, C. Navau, A. Sanchez, J. I. Cirac, Phys. Rev. Lett. 109, 147205 (2012).
[26] D. Kleckner, I. Pikovski, E. Jeffrey, L. Ament, E. Eliel, J. van den Brink and D. Bouwmeester, New Journal of Physics 10, 095020 (2008).

[27] M. Geiselmann, M. L. Juan, J. Renger, J. M. Say, L. J. Brown, F. J. García de Abajo, F. Koppens, and R. Quidant, Nature Nanotechnology 8, 175 (2013).

[28] V. R. Horowitz, B. J. Aleman, D. J. Christle, A. N. Cleland, and D. D. Awschalom, Proc. Natl. Acad. Sci. USA 109, 13493 (2012).

[29] A. Laraoui, J. S. Hodges, and C. A. Meriles, Nano Lett. 12, 3477 (2012).

[30] T. Li, S. Kheifets, and M. G. Raizen, Nat. Phys. 7, 527530 (2011).

[31] J. Gieseler, B. Deutsch, R. Quidant, and L. Novotny, Phys. Rev. Lett. 109, 103603 (2012).

[32] B. Naydenov, F. Dolde, L. T. Hall, C. Shin, H. Fedder, L. C. L. Hollenberg, F. Jelezko, and J. Wrachtrup, Phys. Rev. B 83, 081201 (2011) ; K. D. Jahnke, B. Naydenov, T. Teraji, S. Koizumi, T. Umeda, J. Isoya, and F. Jelezko Applied Physics Letters 101, 012405 (2012); P. L. Stanwix, L. M. Pham, J. R. Maze, D. Le Sage, T. K. Yeung, P. Cappellaro, P. R. Hemmer,A. Yacoby, M. D. Lukin, and R. L. Walsworth, Phys. Rev. B 82, 201201(R) (2010); P. C. Maurer, G. Kucsko, C. Latta, L. Jiang, N. Y. Yao, S. D. Bennett, F. Pastawski, D. Hunger, N. Chisholm, M. Markham, D. J. Twitchen, J. I. Cirac, and M. D. Lukin, Science 336, 1283 (2012); H. Bernien, B. Hensen, W. Pfaff, G. Koolstra, M. S. Blok, L. Robledo, T. H. Taminiau, M. Markham, D. J. Twitchen, L. Childress, and R. Hanson, Nature 497, 86 (2013); M. V. Gurudev Dutt, L. Childress, L. Jiang, E. Togan, J. Maze, F. Jelezko, A. S. Zibrov, P. R. Hemmer, M. D. Lukin, Science 316, 1312 (2007).

[33] A. I. Bishop and P. F. Barker, Rev. Sci. Instrum. 77 , 044701 (2006).

[34] G. Vacanti, R. Fazio, M. S. Kim, G. M. Palma, M. Paternostro, and V. Vedral, Phys. Rev. A 85, 022129 (2012).

[35] T. P. Spiller, K. Nemoto, S. L. Braunstein, W. J. Munro, P. van Loock, G. J. Milburn New J. Phys. 8, 30 (2006); T. A. Brun, Hsi-Sheng Goan, Phys. Rev. A 68, 032301 (2003); L. Garcia, R. W. Chhajlany, Y. Li, Lian-Ao Wu, arXiv:1305.0290 Y. Li, L. Wu, Y. Wang, and L. Yang, Phys. Rev. B 84, 094502 (2011). 